RHIC/AP/96

\title{
Beam Reconstruction in Longitudinal Phase Space
}

\author{
Vibha Mane
}

July, 1996 


\section{Contents}

1 Introduction $\quad 2$

2 Radon Transform $\quad 2$

3 Reconstruction Algorithms

3.1 Iterative Algorithms $\ldots \ldots \ldots \ldots \ldots \ldots \ldots \ldots \ldots \ldots$

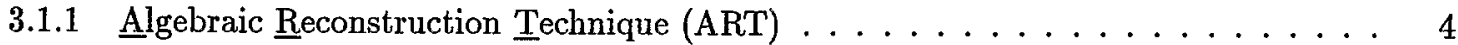

3.1 .2 Least Square Methods . . . . . . . . . . . . . . . . . . . . . . 4

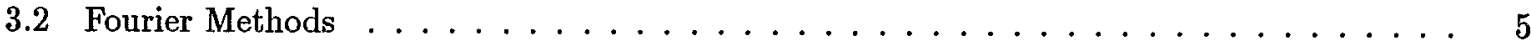

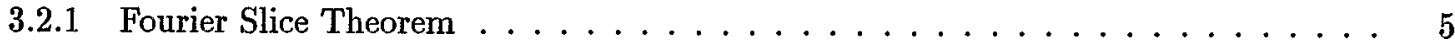

3.2 .2 Filtered backprojection $\ldots \ldots \ldots \ldots \ldots \ldots \ldots$

4 Program Usage $\quad 7$

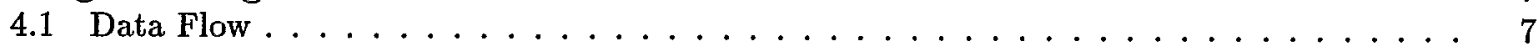

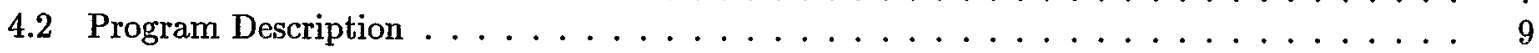

4.2 .1 Profile Generation . . . . . . . . . . . . . . . . . . . 9

4.2 .2 Image Reconstruction and Comparison . . . . . . . . . . . . . . . . 9

4.2 .3 Graphics Display . . . . . . . . . . . . . . . . . . . . 10

5 Examples $\quad 10$

6 Sensitivity Plots $\quad 23$

7 TIBETAN Example $\quad$ • 27

8 AGS Example $\quad 27$

9 Conclusion and Status $\quad 27$

$\begin{array}{ll}10 \text { Acknowledgements } & \mathbf{2 7}\end{array}$ 


\section{Introduction}

Tomograhic techniques are used to reconstruct two dimensional longitudinal phase space plot of the beam from one dimensional profiles. Input data consists of several projections of the beam distribution, over half a synchrotron period. This data is obtained from a wall current monitor.

A program trc (Tomographic Reconstruction) has been written in $\mathrm{C}$ to do the image reconstruction. trc and other codes to generate profiles, read and write profiles in sds format, do graphics display and image comparison are located in $\$$ RADON/docs (Section 4.2 ).

This paper describes the reconstruction algorithm used in trc, program usage and gives some examples. Also given are sensitivity plots, which show the error in reconstruction due to insufficient profiles, samples, random noise, and an error estimate in synchrotron period.

\section{Radon Transform}

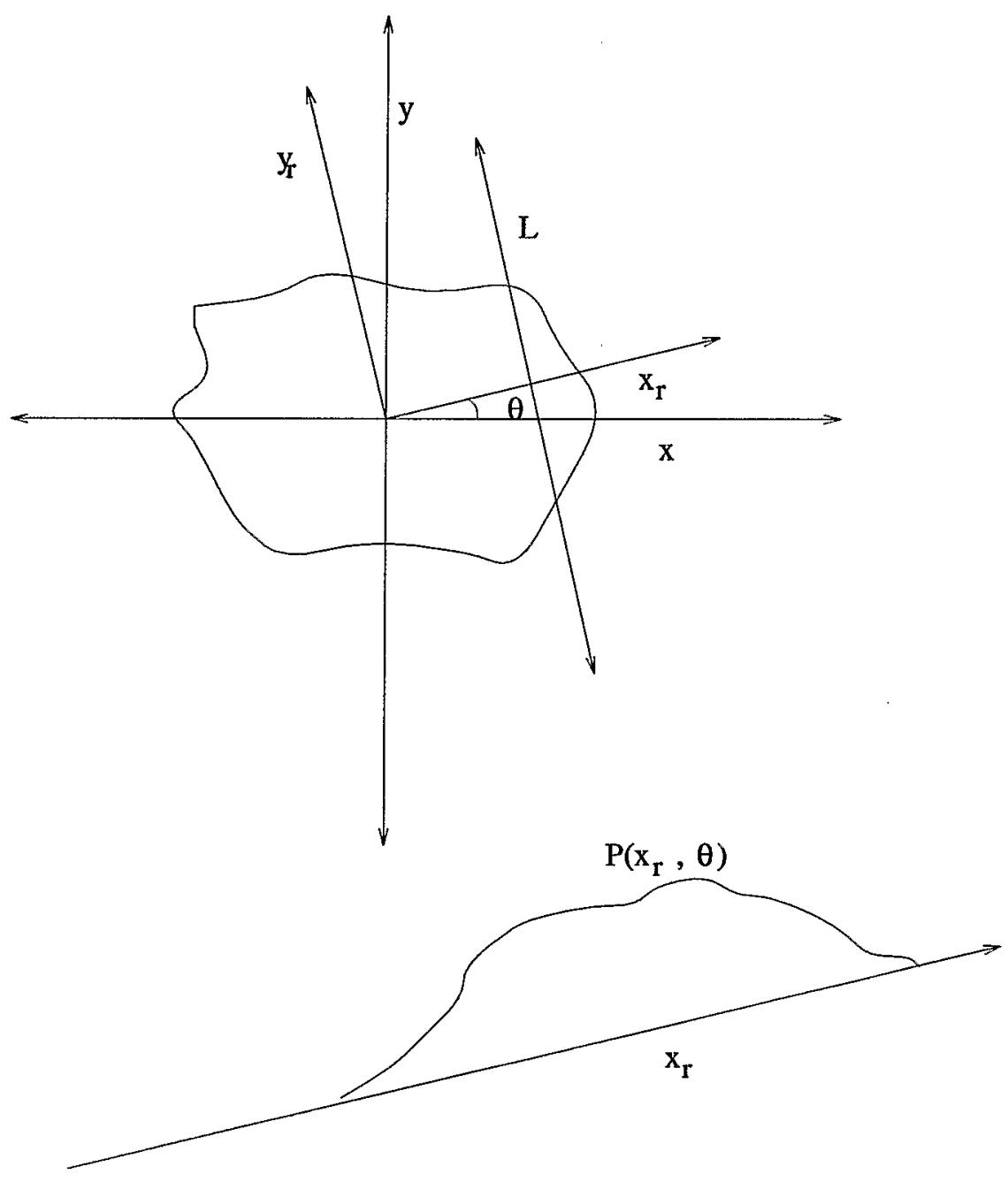

Figure 1: Radon Transform of a two dimensional object 
Let $f(x, y)$ represent a 2D object (Figure 1). Let $\left(x_{r}, y_{r}\right)$ be the coordinate system rotated by angle $\theta$ with respect to the $(\mathrm{x}, \mathrm{y})$ coordinate system, where $x_{r}=x \cos \theta+y \sin \theta$ and $y_{r}=-x \sin \theta+y \cos \theta$. Let $\mathrm{L}$ be any line in the $(\mathrm{x}, \mathrm{y})$ plane. The projections $P\left(x_{r}, \theta\right)$ obtained by integrating $\mathrm{f}(\mathrm{x}, \mathrm{y})$ along all possible lines $L$ is the Radon transform of $f(x, y)$ [1] [2].

$$
P\left(x_{r}, \theta\right)=\int_{-\infty}^{\infty} f\left(x_{r}, y_{r}\right) d y_{r}
$$

These projections are taken for several values of $\theta$ between 0 and $\pi$. The $2 \mathrm{D}$ function $\mathrm{f}(\mathrm{x}, \mathrm{y})$ can then be obtained from the profiles using inversion techniques described below.

In longitudinal phase space, particles orbit around the synchronous particle, in the rf bucket. Synchrotron oscillation time is typically several hundred turns. The wall current monitor, which is a broadband current transformer, gives the bunch profile with time as the bunch passes by. Therefore many bunch profiles can be measured over half a synchrotron period, and hence the bunch can be reconstructed in longitudinal phase space.

\section{Reconstruction Algorithms}

The reconstruction algorithms can be broadly classified into Iterative Algorithms and Fourier Methods [3] [4]:

\subsection{Iterative Algorithms}

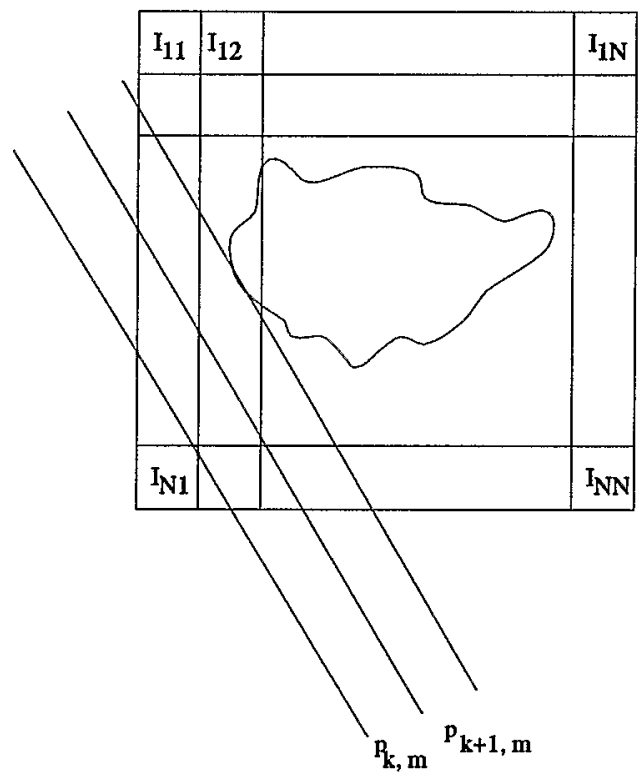

Figure 2: Image on a two dimensional grid

Let the image be represented on a square grid of size $N \times N$. Let $I_{i j} \equiv f(x, y)$ in cell $(i, j)$. Let $p_{k m}$ represent the projection for $\mathrm{kth}$ bin and mth angle (Figure 2).

$$
\sum_{i, j} w_{i j}^{k m} I_{i j}=p_{k m}
$$


i: $1 \ldots \mathrm{N}, \mathrm{j}: 1 \ldots \mathrm{N}, \mathrm{k}: 1 \ldots \mathrm{N}, \mathrm{m}: 1 \ldots \mathrm{M}, \mathrm{M}=$ number of projections.

$w_{i j}^{k m}$ - weight matrix. It is the contribution to the projection $p_{k m}$ from cell $(\mathrm{i}, \mathrm{j})$.

\subsubsection{Allgebraic eeconstruction Technique (ART)}

This algorithm is described by R. Gordon et. al. [5] [6] and G. T. Herman et. al. [7].

Let $X_{i j}$ be the image to be reconstructed.

Make an initial guess for $X_{i j}, X_{i j}^{0}=0$

At each iteration, find the difference between the measured projection, and the projection from the image under construction, and apply the appropriate correction.

$$
X_{i j}^{q+1}=X_{i j}^{q}+\frac{w_{i j}^{k m}\left(p_{k m}-r_{k m}^{q}\right)}{W_{k m}}
$$

$\mathrm{q}$ - iteration number, $p_{k m}$ - measured projections

$$
\begin{gathered}
r_{k m}^{q}=\sum_{k, m} X_{i j}^{q} w_{i j}^{k m} \\
W_{k m}=\sum_{i, j} w_{i j}^{k m}
\end{gathered}
$$

\section{Constraints}

The reconstruction can be partially or fully constrained as follows: $X_{i j}>0$ and $X_{i j}<I_{\max }$, where $I_{\max }$ is the known maximum value of $I_{i j}$.

Convergence Criteria

ART converges to a solution which minimizes discrepancy, variance and entropy defined as follows:

Discrepancy

$$
D^{q}=\sqrt{\frac{1}{M N} \sum_{k m} \frac{\left(p_{k m}-r_{k m}\right)^{2}}{W_{k m}}}
$$

Variance

$$
V^{q}=\sum_{i j}\left(X_{i j}-\bar{X}\right)^{2}
$$

Entropy

$$
S^{q}=\frac{-1}{\ln N^{2}} \sum_{i j} \frac{X_{i j}^{q}}{\bar{X}} \ln \frac{X_{i j}^{q}}{\bar{X}}
$$

$\bar{X}$ is the average value of the image cells.

\subsubsection{Least Square Methods}

This method minimizes the difference between the measured projections and the projections of the reconstructed image using an optimization algorithm such as conjugate gradient method or the steepest descent method. This algorithm is described in more detail in [8]. 


\subsection{Fourier Methods}

These algorithms are described by Bracewell and Riddle [10], Ramachandran and Lakshminarayanan [11].

\subsubsection{Fourier Slice Theorem}

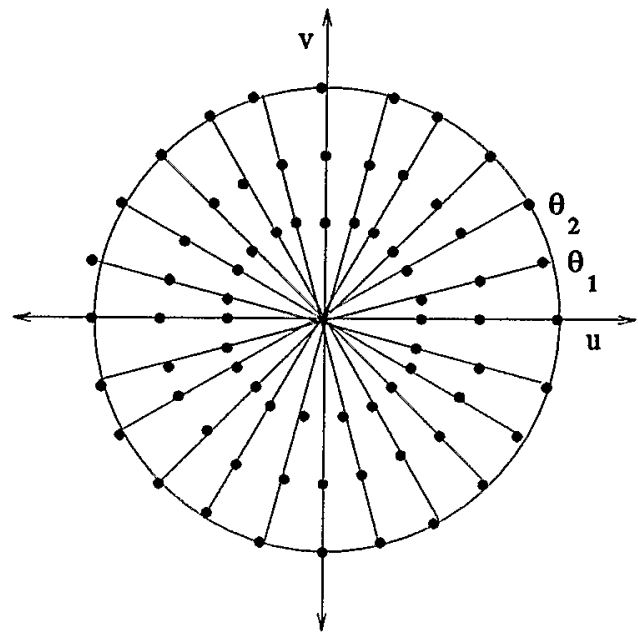

Figure 3: Slices of the Fourier Transform $F(u, v)$ of the original object $f(x, y)$

Let $F(u, v)$ be the 2-dimensional Fourier Transform of $f(x, y)$. The Fourier Transform of a projection at angle $\theta$ wrt $\mathrm{x}$ gives a slice of the 2D Fourier Transform $\mathrm{F}(\mathrm{u}, \mathrm{v})$ at angle $\theta$ wrt $\mathrm{u}$, Figure 3. Therefore by taking projections at angles $\theta_{1}, \theta_{2} \ldots$ and Fourier transforming each projection, we have $F(w, \theta)$ along radial lines, where $u=w \cos \theta$ and $v=w \sin \theta . F(w, \theta)$ is interpolated from a polar grid to obtain $\mathrm{F}(\mathrm{u}, \mathrm{v})$ on a square grid. The inverse Fourier Transform of $F(u, v)$ gives $f(x, y)$.

\subsubsection{Filtered backprojection}

The filtered backprojection algorithm is used in trc. Figure 4 illustrates backprojection and superposition. In this algorithm, each projection is filtered with a high pass filter, the filtered projections are backprojected and summed up.

$F(u, v)$ is the 2D Fourier Transform of $f(x, y)$.

$$
\begin{gathered}
f(x, y)=\int_{-\infty}^{\infty} \int_{-\infty}^{\infty} F(u, v) e^{i 2 \pi(u x+v y)} d u d v \\
u=w \cos \theta \quad v=w \sin \theta \\
f(x, y)=\int_{0}^{2 \pi} \int_{0}^{\infty} F(w, \theta) e^{i 2 \pi w(x \cos \theta+y \sin \theta)} w d w d \theta \\
f(x, y)=\int_{0}^{\pi} \int_{-\infty}^{\infty} F(w, \theta)|w| e^{i 2 \pi w x_{r}} d w d \theta \\
x_{r}=x \cos \theta+y \sin \theta \quad F(w, \theta+\pi)=F(-w, \theta) \quad F(w, \theta) \equiv S_{w}(\theta)
\end{gathered}
$$



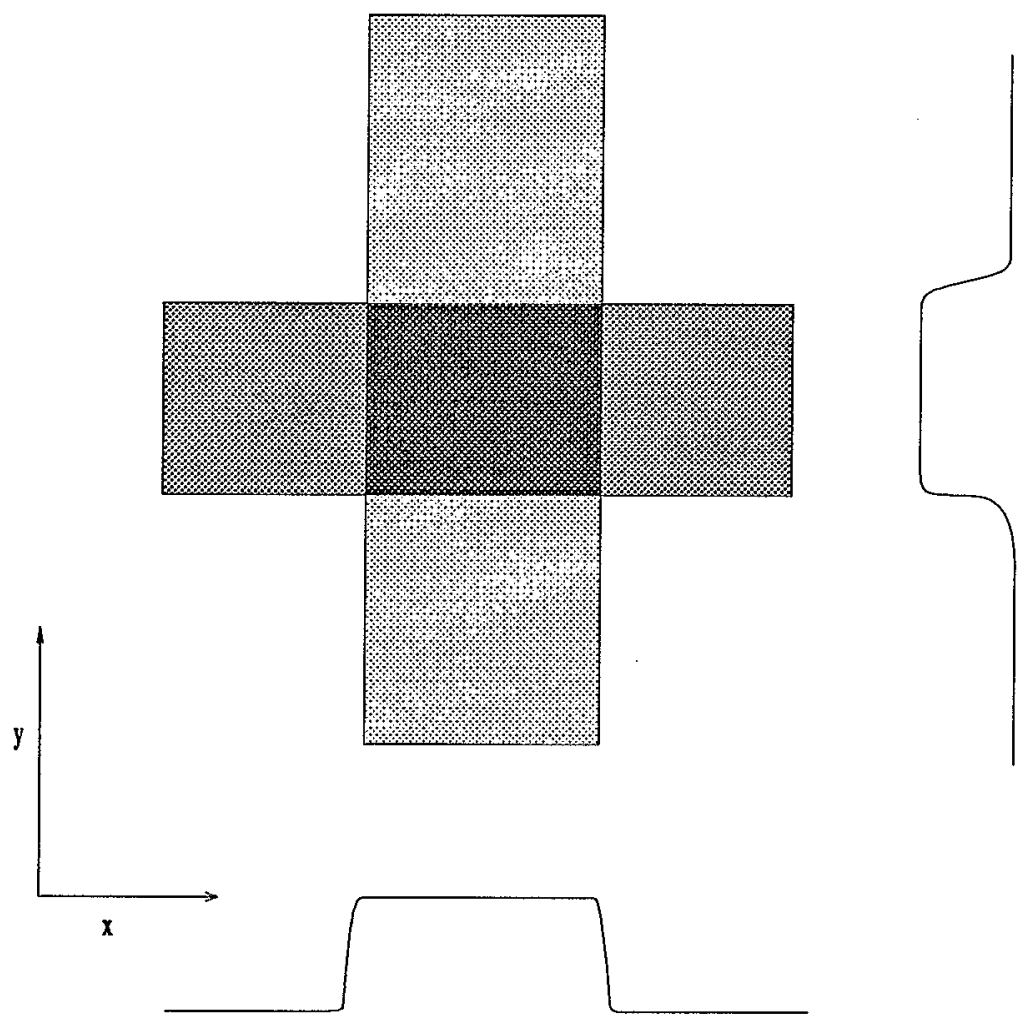

Figure 4: Example of backprojection and superposition

$$
f(x, y)=\int_{0}^{\pi} \int_{-\infty}^{\infty} S_{w}(\theta)|w| e^{i 2 \pi w x_{r}} d w d \theta
$$

$S_{w}(\theta)$ is the Fourier Transform of $P\left(x_{r}, \theta\right)$.

$$
Q\left(x_{r}, \theta\right)=\int_{-\infty}^{\infty} S(w, \theta)|w| e^{i 2 \pi w x_{r}} d w
$$

is the filtered projection.

The reconstructed image can be obtained from

$$
f(x, y)=\int_{0}^{\pi} Q\left(x_{r}, \theta\right) d \theta
$$

The following two filters are defined in trc [8]

1. Ramp Filter

$$
\begin{aligned}
& H(f)= \begin{cases}|f| & \text { if }|f| \leq f_{c} \\
0 & \text { otherwise }\end{cases} \\
& f_{c}=\text { cutoff frequency }
\end{aligned}
$$




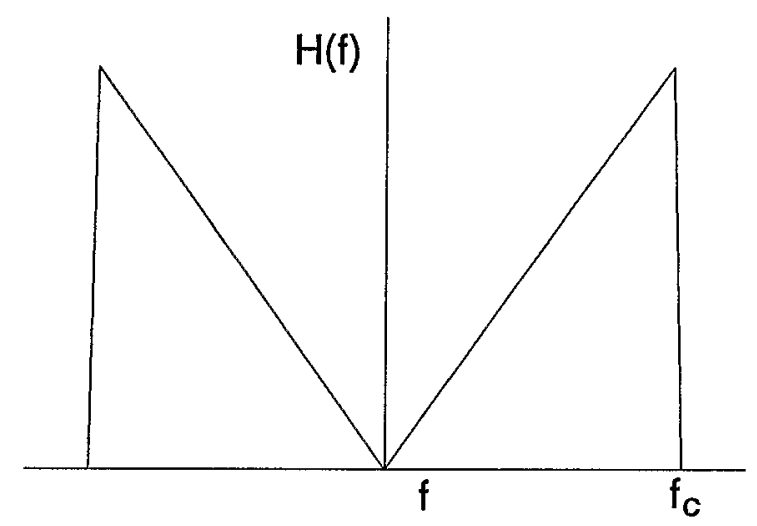

Figure 5: Ramp filter

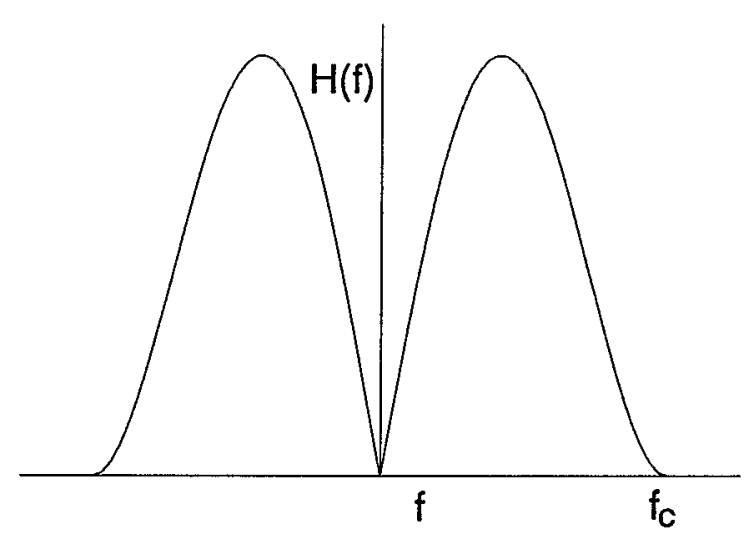

Figure 6: Hann filter

2. Hann Filter

$$
H(f)= \begin{cases}0.5|f|\left(1+\cos \left(\pi f / f_{c}\right)\right) & \text { if }|f| \leq f_{c} \\ 0 & \text { otherwise }\end{cases}
$$

In future upgrades the Hamming and the Butterworth filters will also be implemented.

\section{Program Usage}

\subsection{Data Flow}

Figure 7 shows the data flow. Mountain range data from the AGS wall current monitor is captured into a fast digital scope, LeCroy Model 9354TM. The data is transfered to the computer, via GPIB using a LabVIEW program [16] [17]. This data is then converted to the sds format using program 


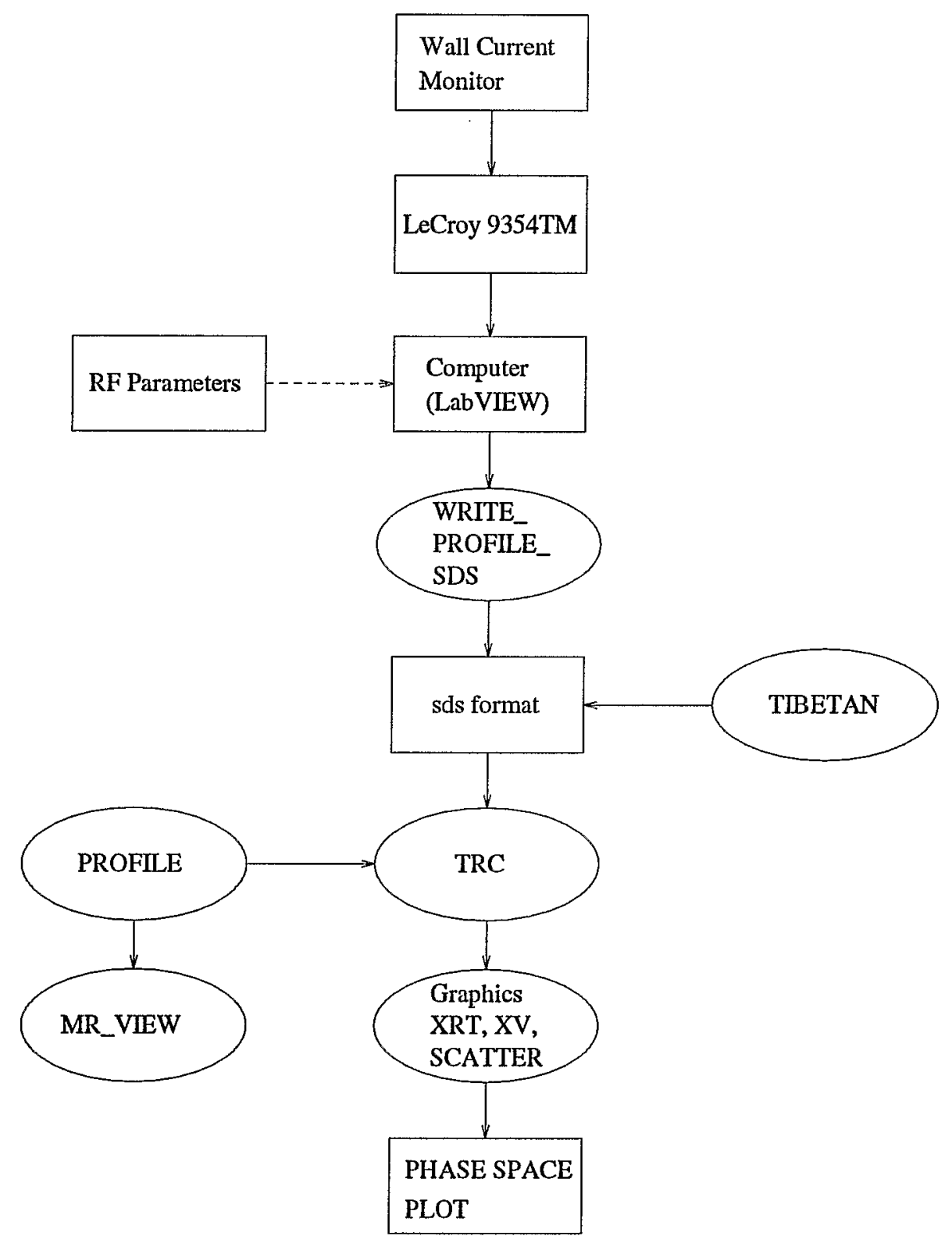

Figure 7: Data Flow for Reconstruction 
write_profile_sds. trc reads this data in sds format, reconstructs the image, and writes it to an ascii file. Also required are the RF parameters from the machine, so that the image is written in appropriate longitudinal phase space coordinates $\Delta p / p$ vs. $\Delta \phi$. The mountain range data flow is automated, but the $\mathrm{RF}$ parameter data flow is not yet automatic.

trc also reads output data from tibetan [14], in the sds format. For more on tibetan, please see Section 7. The program profle generates test profiles for a few beam distributions such as gaussian, annular, exponential and rectangular. The output data is written in ascii format, and read into trc. This is used mainly to test trc. The program mr-view can be used to plot the profiles as mountain range display. The output image from trc can be plotted using any one of the grahics display programs, such. as $x v, x r t$ and scatter.

\subsection{Program Description}

A package of codes has been developed in $\mathrm{C}$ and $\mathrm{C}++$ to generate profiles, reconstruct images and display them. The code is written in C, mainly so that it would read data in the standard sds [13] format. Given below is a description of the various programs, in the package. For Program Usage and Environment Setup, source /usr/publiv/ENV/setup-radon, and see README in \$RADON/docs.

\subsubsection{Profile Generation}

profile

Generates profiles for beam distributions such as gaussian, annular, exponential and rectangular. Input parameters such as number of profiles, number of samples (points per profile), density distribution, image window size, and noise level can be selected. The output data is written in ascii format, and can be viewed with $x m g r$. This data can be used as a test input to trc. profile also writes the original image, which can be used for comparison with the reconstructed image.

write_profile_sds

Reads the machine parameters and experimental data from LabVIEW, and writes it in sds format.

mr_view

The output data from profile is written in a format so that it can be displayed as a mountain range plot, using $x m g r$.

\subsubsection{Image Reconstruction and Comparison}

tre

Reads input profiles in sds or ascii format, does the image reconstruction, and writes the image intensity vs. $\mathrm{x}$ and $\mathrm{y}$. If the input is in sds format, with the rf parameters known, the image is written in longitudinal phase space coordinates $\Delta p / p$ vs. $\Delta \phi$. Input parameters such as the type of filter, cutoff frequency, read format etc. can be selected.

image_comp

Compares the reconstructed image with the original image, if available, and gives the error in reconstruction as defined in Section 6

image_intr 
Reads an input image, and creates an output image of a different specified size, using bilinear interpolation.

\subsubsection{Graphics Display}

$c x v$

Reads the output image from $t r c$, and writes it in $x v$ format, which can be displayed using $x v$.

cxrt

Reads the output image from $t r c$, and writes it in $x r t$ format, which can be displayed using $x r t$ [15].

scatter

Reads the output image from $t r c$, and writes it in scatter format, which can be plotted with $x m g r$. This can be compared with the scatter plot output from tibetan.

\section{$5 \quad$ Examples}

This section gives some examples of image reconstruction, using trc. The program profile is used to generate profiles for gaussian and annular distributions. The total profiles used are equally spaced between 0 and $\pi$. Also given is the signal and background error, as described below, which compares the original image with the reconstruction. Some other criteria for image comparison are described in [9].

Signal Error

$I_{i j}$ is the intensity in cell $(i, j)$ of the original image, $X_{i j}$ is the intensity in cell $(i, j)$ of the reconstructed image. Let $I_{\max }$ be the maximum intensity in the original image. Define the cutoff intensity $I_{c}$ to be $10 \%$ of the maximum intensity, thet is $I_{c}=0.1 I_{\text {max }}$. Define the signal region $\mathrm{S}$ such that $I_{i j}>I_{c}$. Signal Error is defined by

$$
E_{s}=\frac{1}{I_{\max }-I_{\min }} \sqrt{\frac{1}{\alpha_{s}} \sum_{i, j \in S}\left(I_{i j}-X_{i j}\right)^{2}}
$$

where

$$
\alpha_{s}=\sum_{i, j \in S} 1
$$

Background Error

Define the background region B consisting of all the cells $(i, j)$ that are not in S. Background Error is defined by

$$
E_{b}=\frac{1}{I_{\max }-I_{\min }} \sqrt{\frac{1}{\alpha_{b}} \sum_{i, j \in B}\left(I_{i j}-X_{i j}\right)^{2}}
$$

where

$$
\alpha_{b}=\sum_{i, j \in B} 1
$$

$I_{\max }-I_{\min }=I_{\max }$ if $I_{\max }=I_{\min }$.

Figure 8 compares reconstructed image with the original, for a gaussian distribution. Also plotted are input profiles (Figure 9) used for the reconstruction. The number of profiles used is 30 , and the size of the image is $64 \times 64$. The signal and background errors are less than .01 . 
The phase space reconstruction would be useful if the beam is distorted due to coherent instabilities. The longitudinal coherent bunched beam modes are described by coupled bunch modes and within bunch modes. The azimuthal mode number $l$ gives the number of periods of phase space density modulation in the azimuthal direction, and the radial mode number $m$ gives the number of periods of the phase space density modulation in the radial direction [12]. For an annular distribution, the original and the reconstructed beam are compared for modes $l=0,3$ and 6 . Figure 10 compares the reconstructed image with the original for $l=0$. The size of the image is $64 \times 64$ and the number of input profiles is 30 (Figure 12). The filter used for reconstruction is the hann filter and the signal and background errors are .28 and .11 , respectively. Figure 11 shows the same image reconstructed using the ramp filter, with signal and background errors being .25 and .09 respectively. The errors with the ramp filter are lower, as the image amplitude is closer to the original. However the image quality with the hann filter is better as it helps reduce the background noise in reconstruction.

Figure 13 compares the reconstructed image with the original for $l=3$. The signal and background errors are .21 and .07 , respectively. The input profiles are shown in figure 14. Figure 15 compares the original and the reconstructed image, for $l=6$. The signal and background errors are .21 and .07 . The input profiles are shown in 16. Figure 17 gives the reconstructed image with $20 \%$ random noise in the input profiles (Figure 18). The signal and noise errors are .21 and .10. 

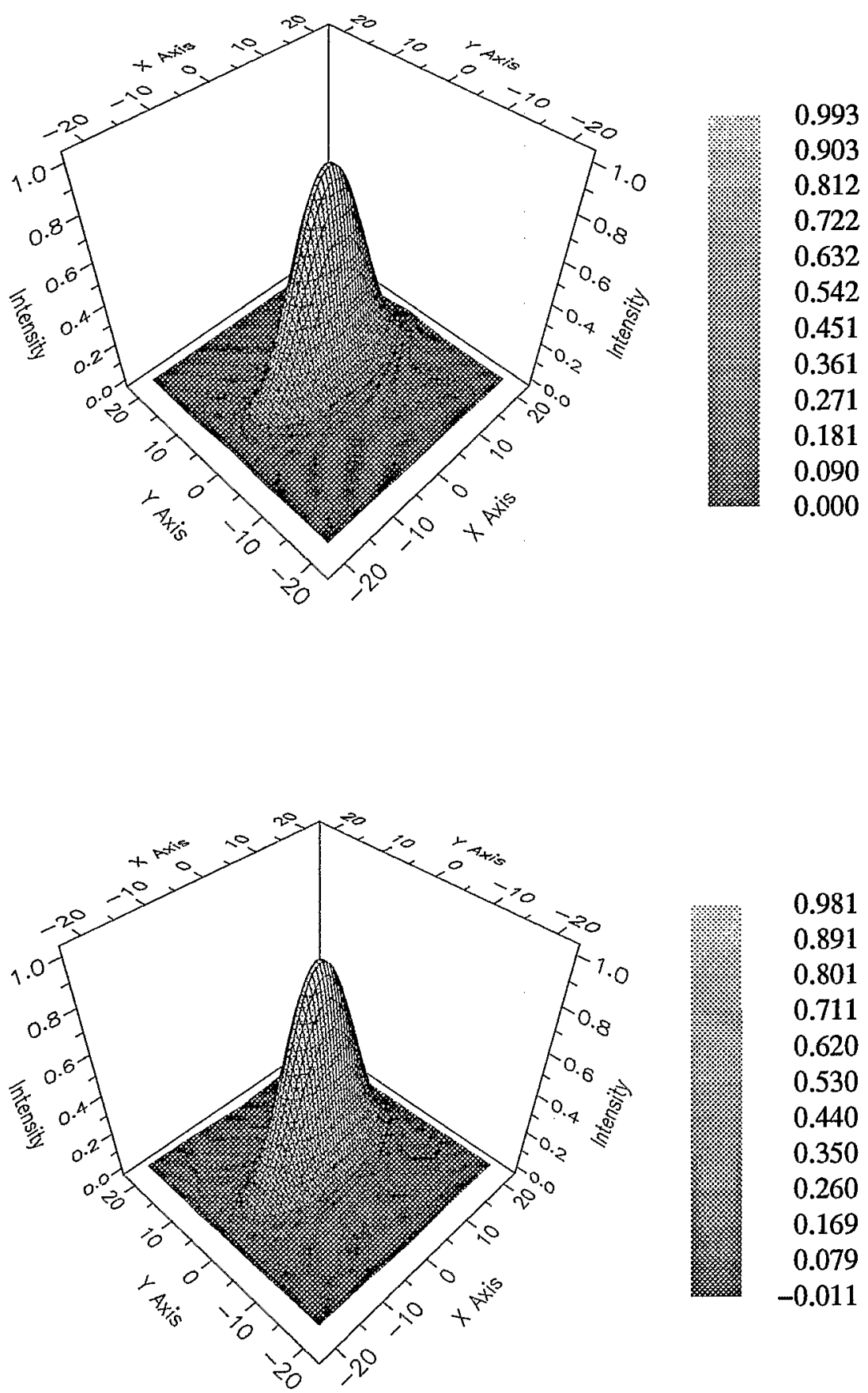

0.981

0.891

0.801

0.711

0.620

0.530

0.440

0.350

0.260

0.169

0.079

$-0.011$

Figure 8: Comparison of the original image (top) with image reconstructed using trc (bottom). Total profiles $=30$, samples $=64$. 


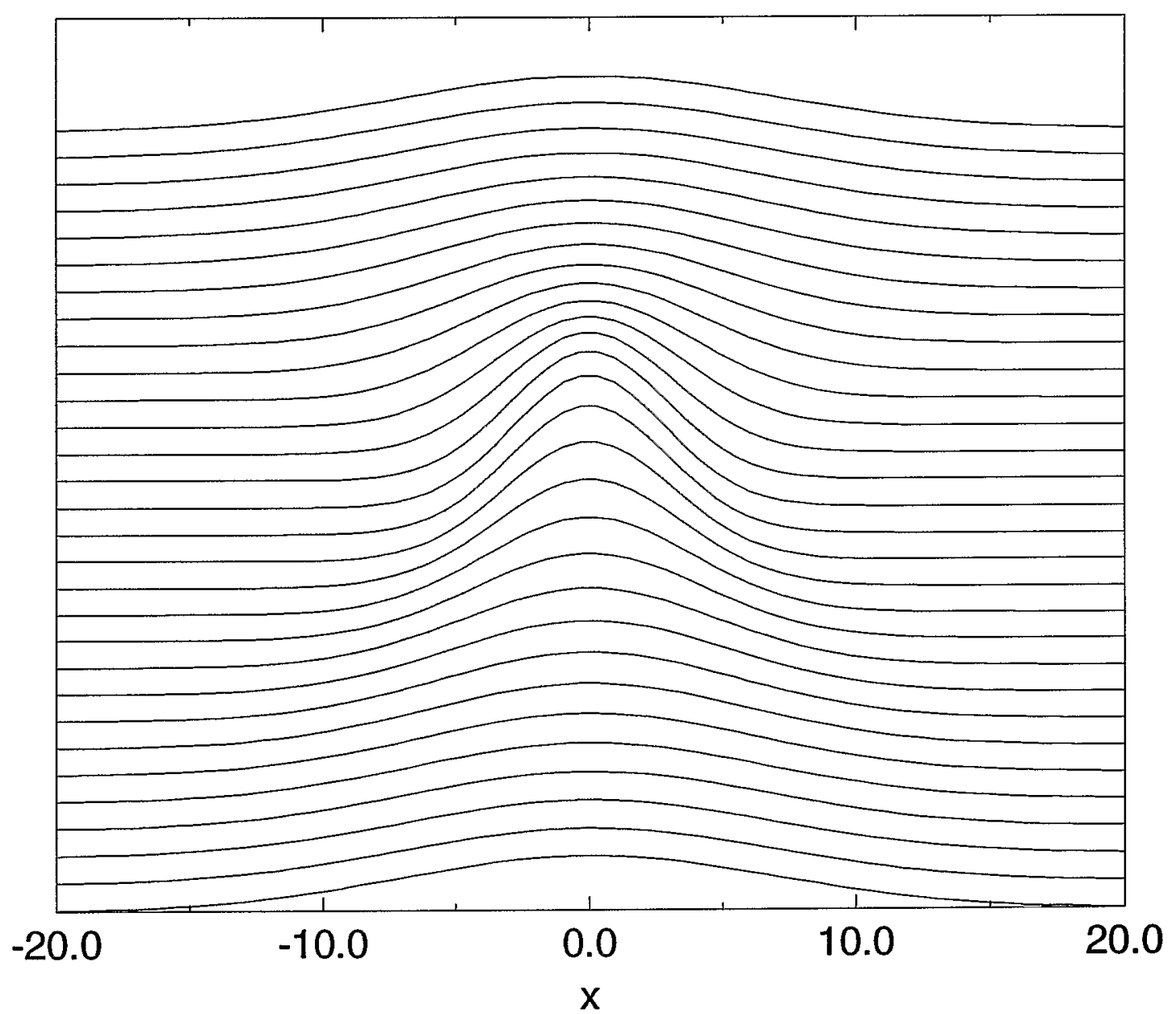

Figure 9: Input profiles for the reconstruction in figure 8. Total profiles $=30$. 

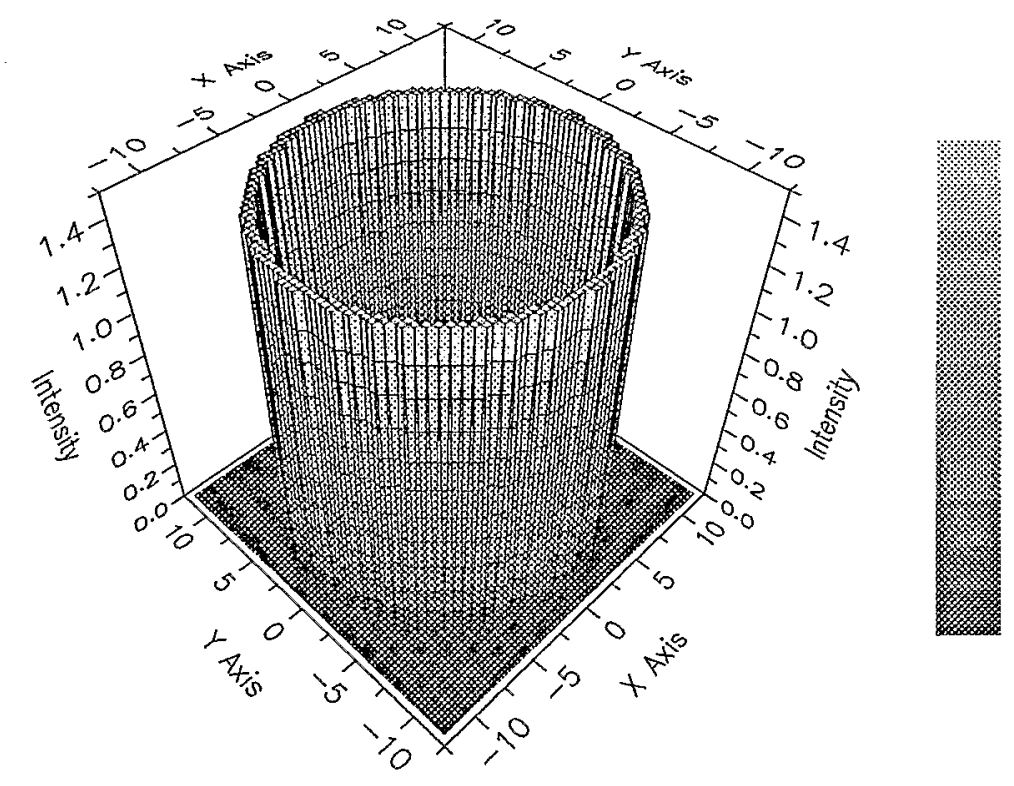

1.50

1.36

1.23

1.09

0.95

0.82

0.68

0.55

0.41

0.27

0.14

0.00

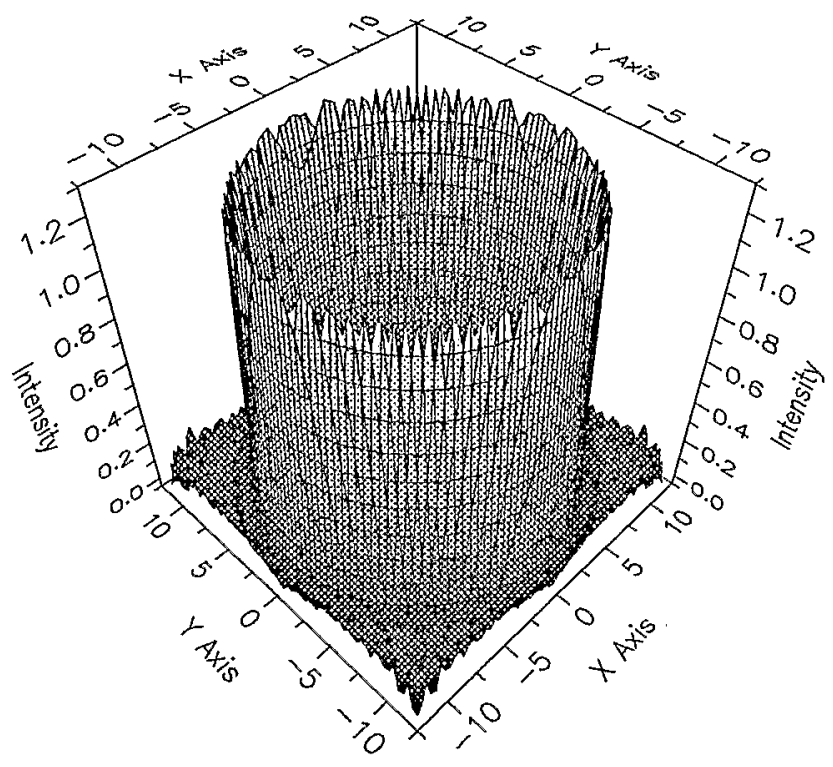

1.30

1.18

1.06

0.94

0.81

0.69

0.57

0.44

0.32

0.20

0.08

$-0.05$

Figure 10: Original image (top) and reconstruction (bottom) using $t r c$, with hann filter. Total profiles = 30 , samples $=64$. Annular beam, azimuthal mode number $\mathrm{l}=0$. 

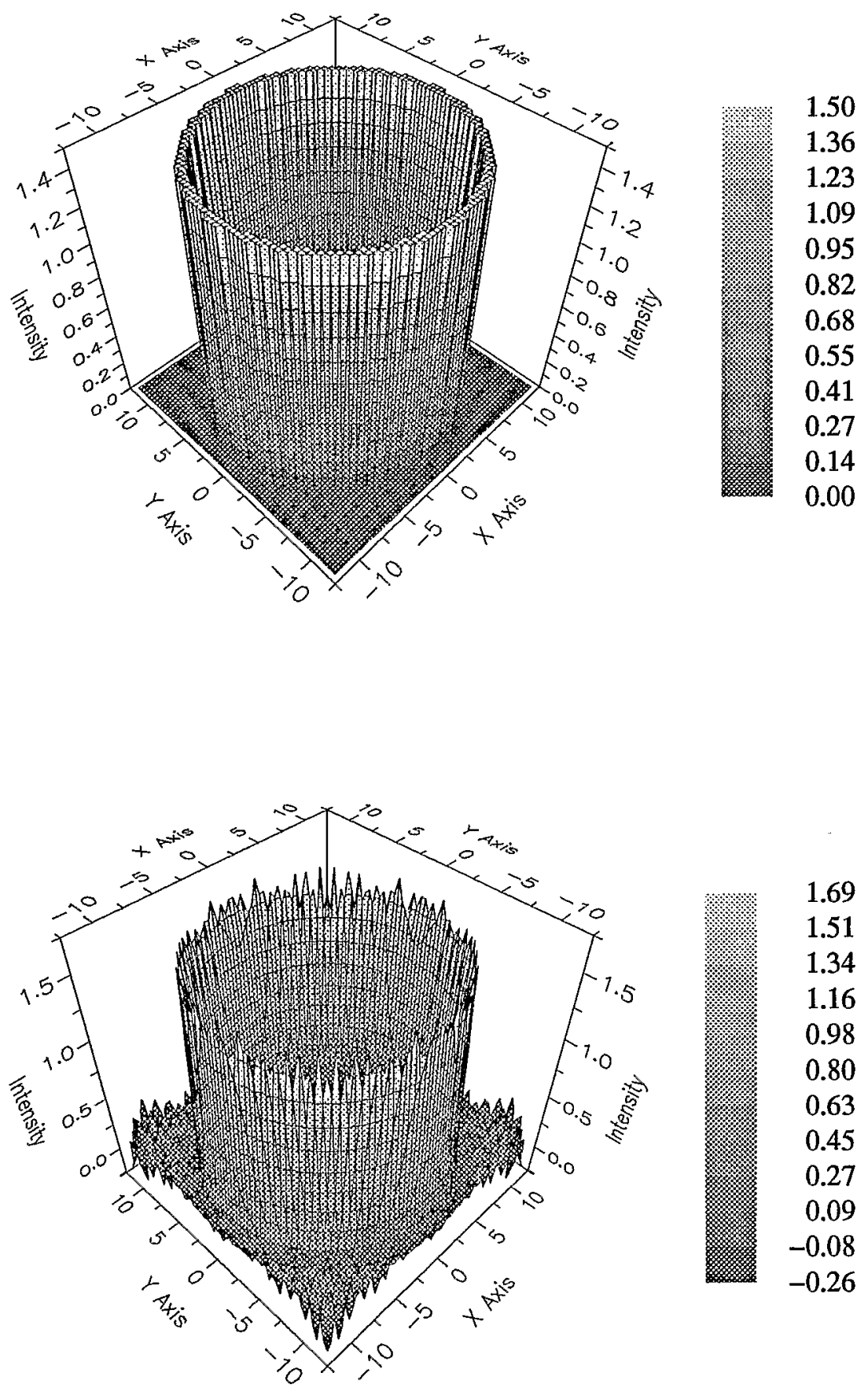

Figure 11: Same as in Figure 10, except the reconstruction is done using $t r c$, with ramp filter. Original image (top) and reconstruction (bottom). The hann filter reduces noise better than the ramp filter. Total profiles $=30$, samples $=64$. Annular beam, azimuthal mode number $l=0$. 


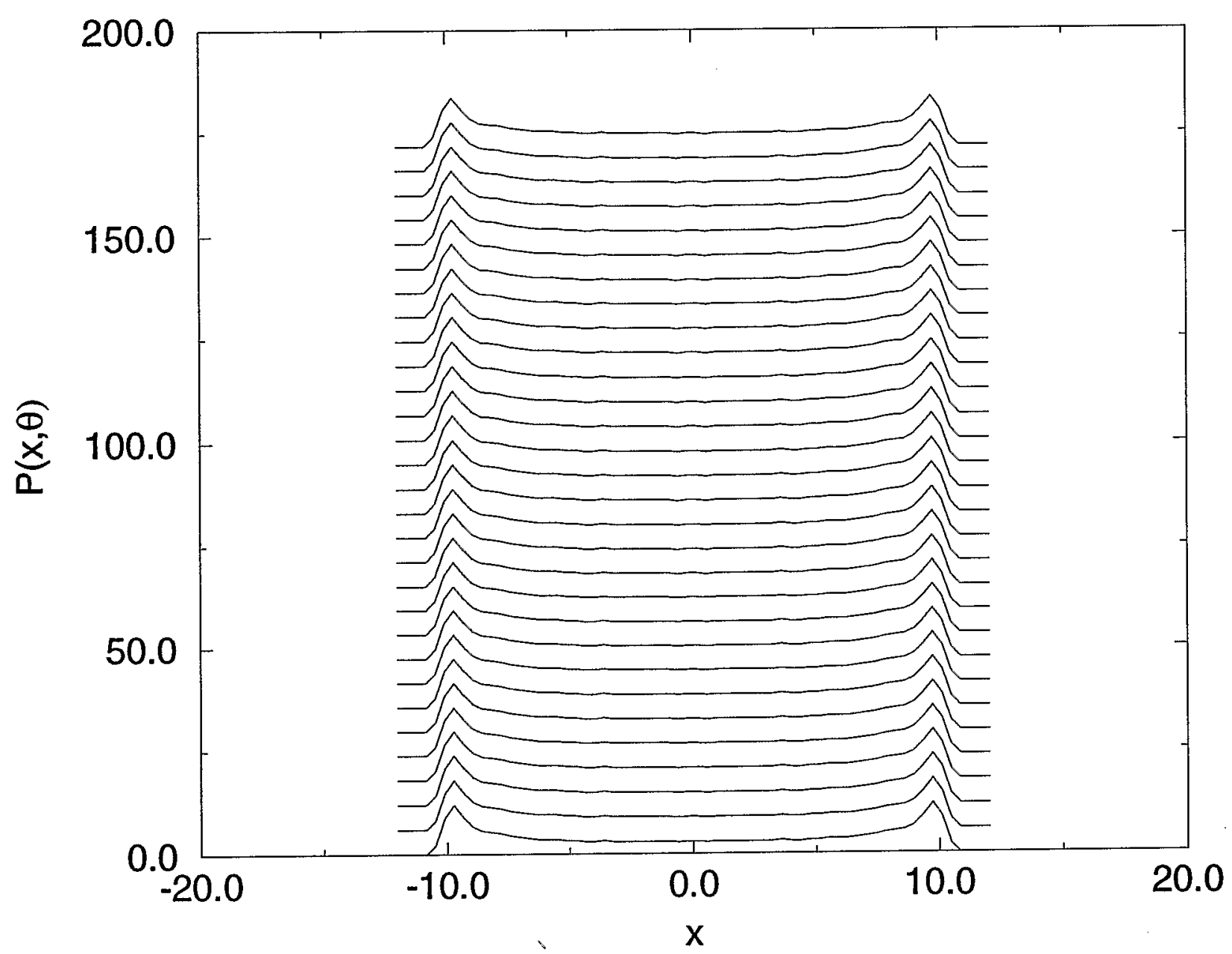

Figure 12: Input profiles for the reconstruction in figures 10 and 11 . Total profiles $=30$. 

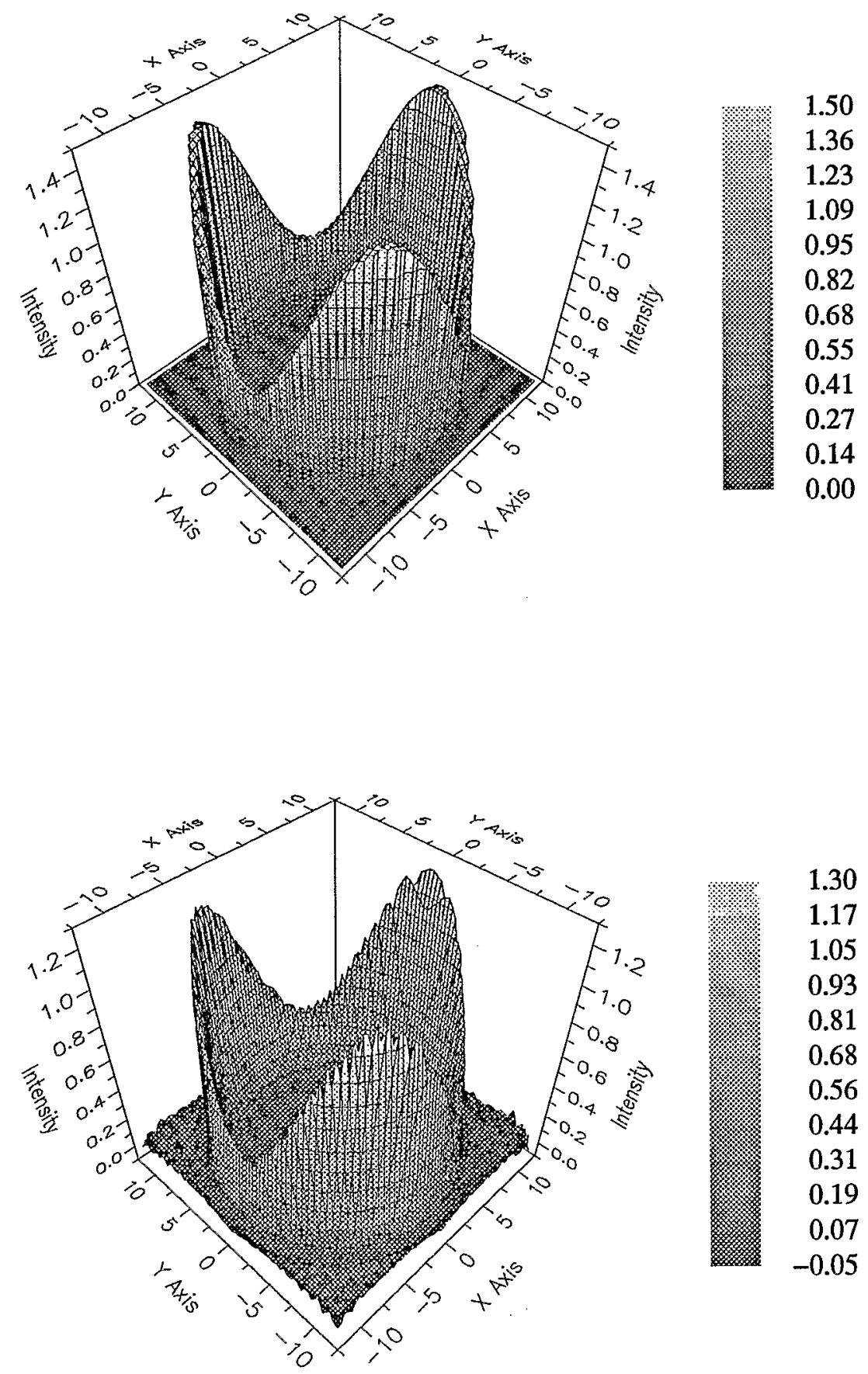

Figure 13: Original image (top) and reconstruction using trc, with hann filter (bottom). Total profiles = 30, samples $=64$. Annular beam, azimuthal mode number $1=3$. 


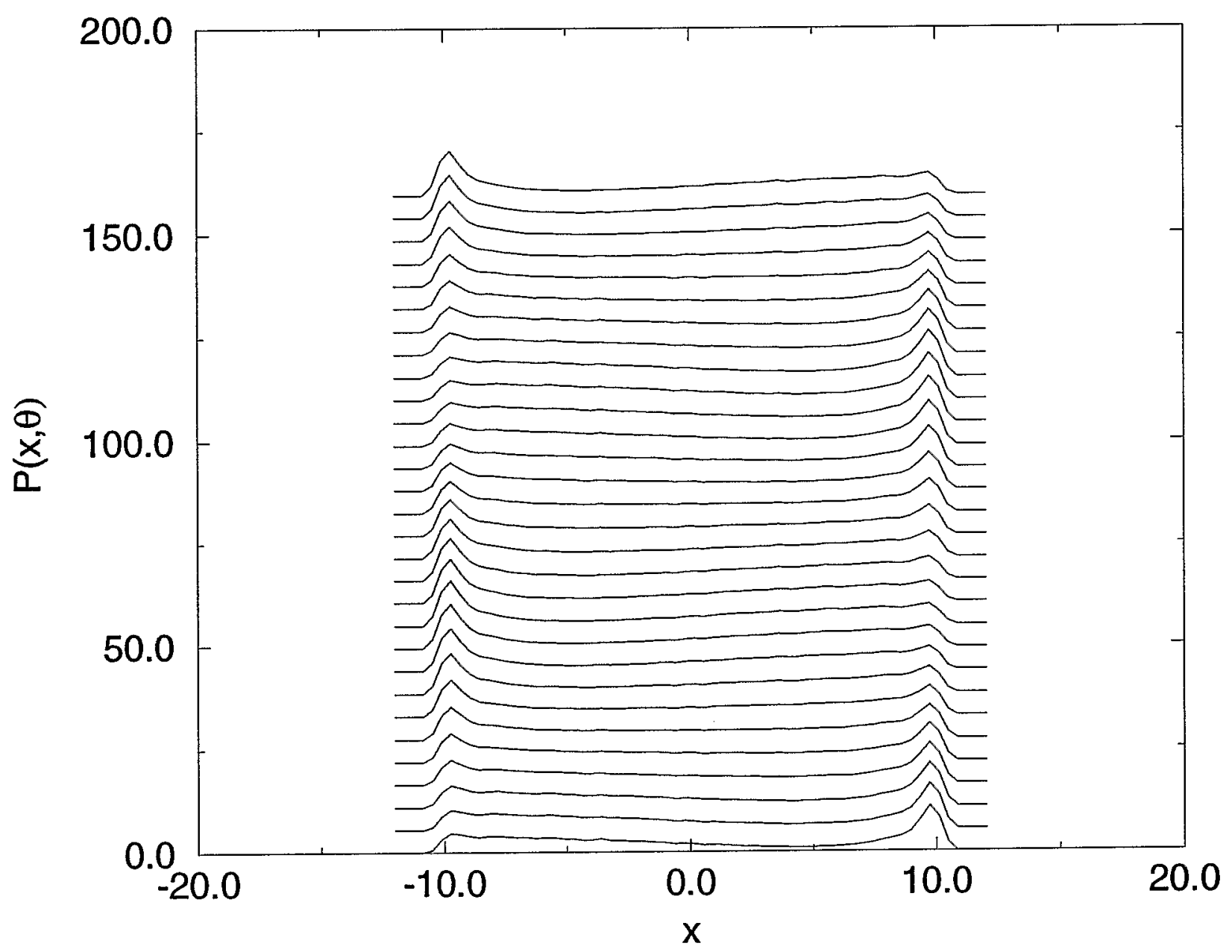

Figure 14: Input profiles for the reconstruction in figure 13. Total profiles $=30$. 

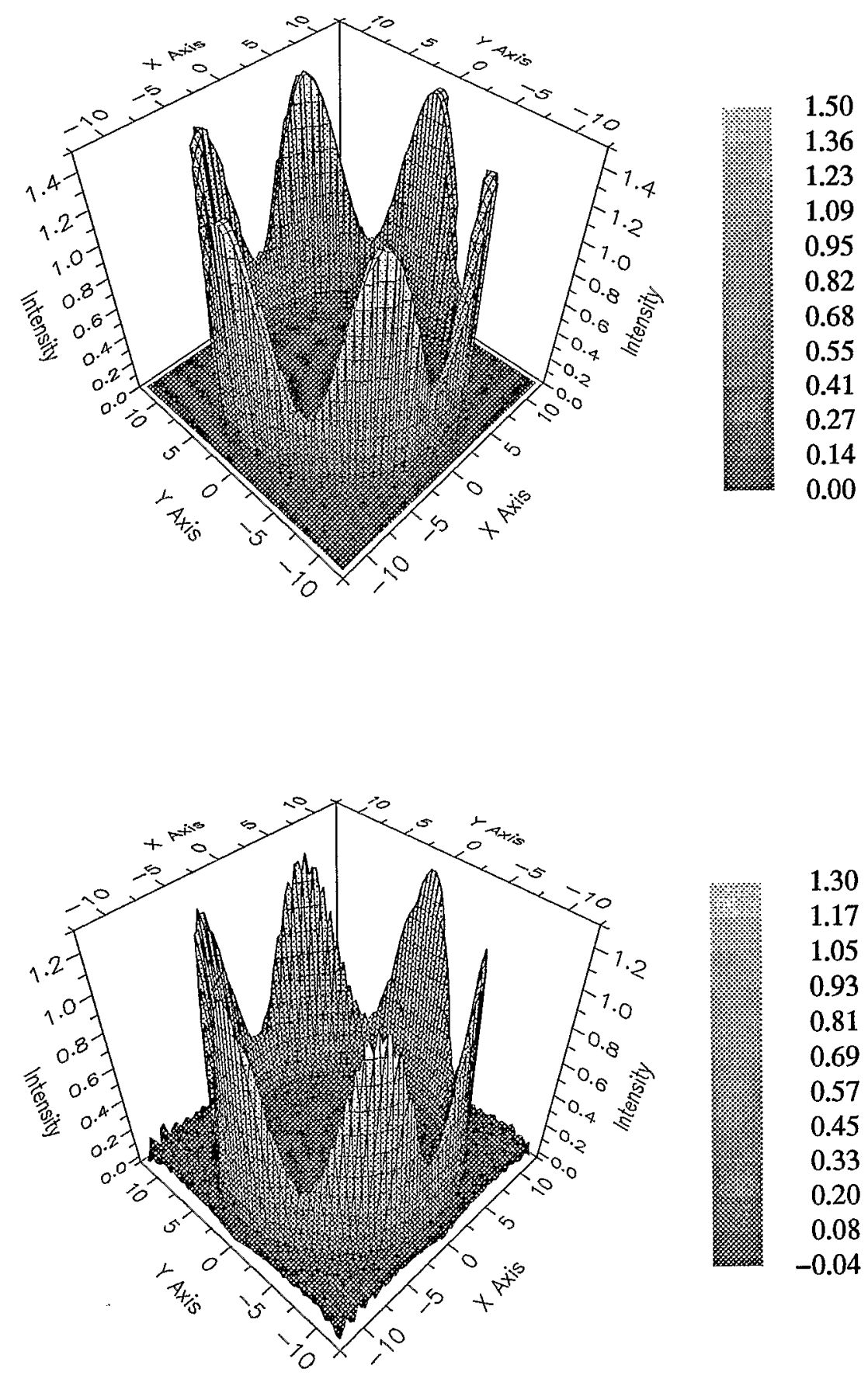

Figure 15: Original image (top) and reconstruction (bottom) using trc, with hann filter. Total profiles $=$ 30 , samples $=64$. Annular beam, azimuthal mode number $l=6$. 


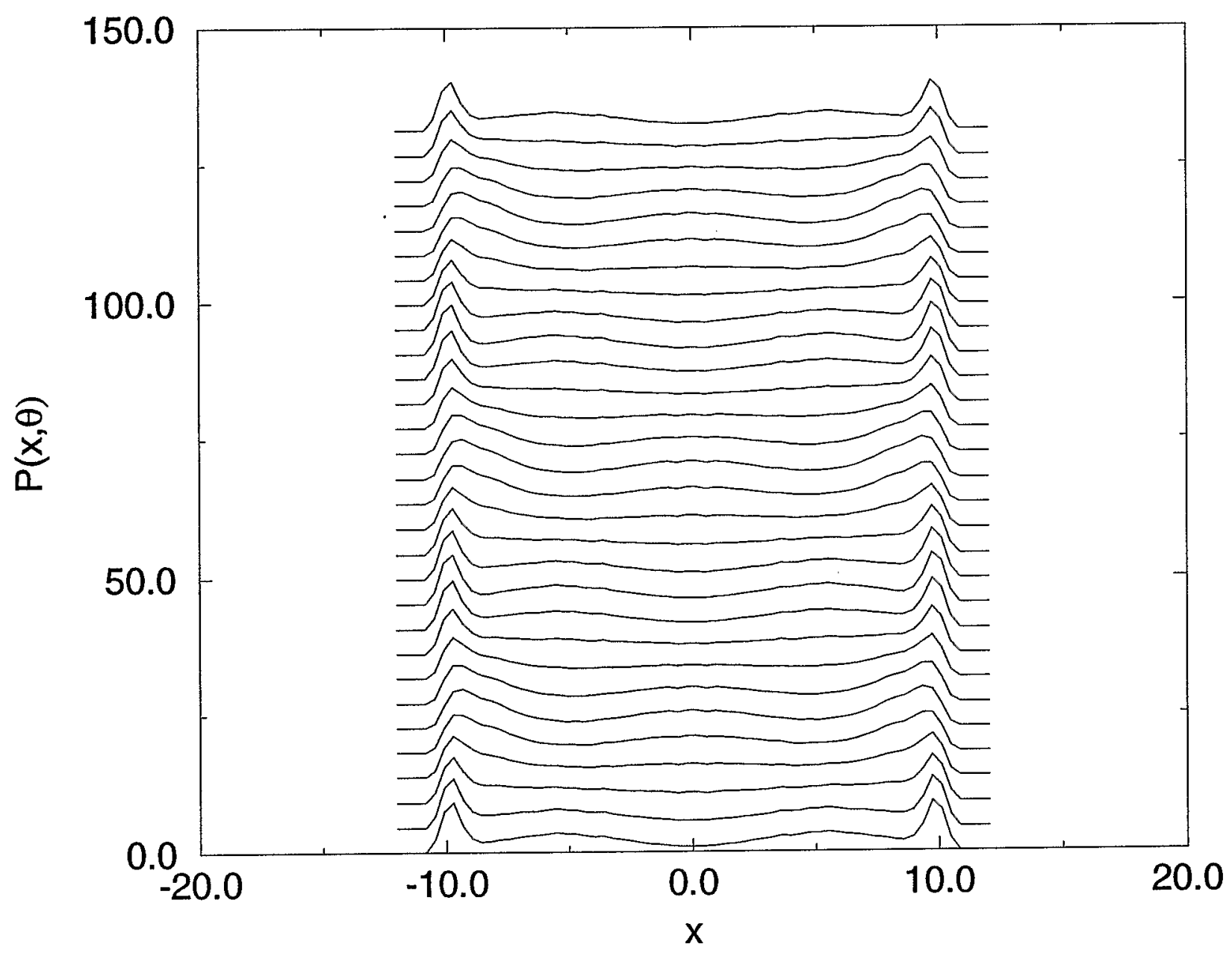

Figure 16: Input profiles for the reconstruction in figure 15. Total profiles $=30$. 

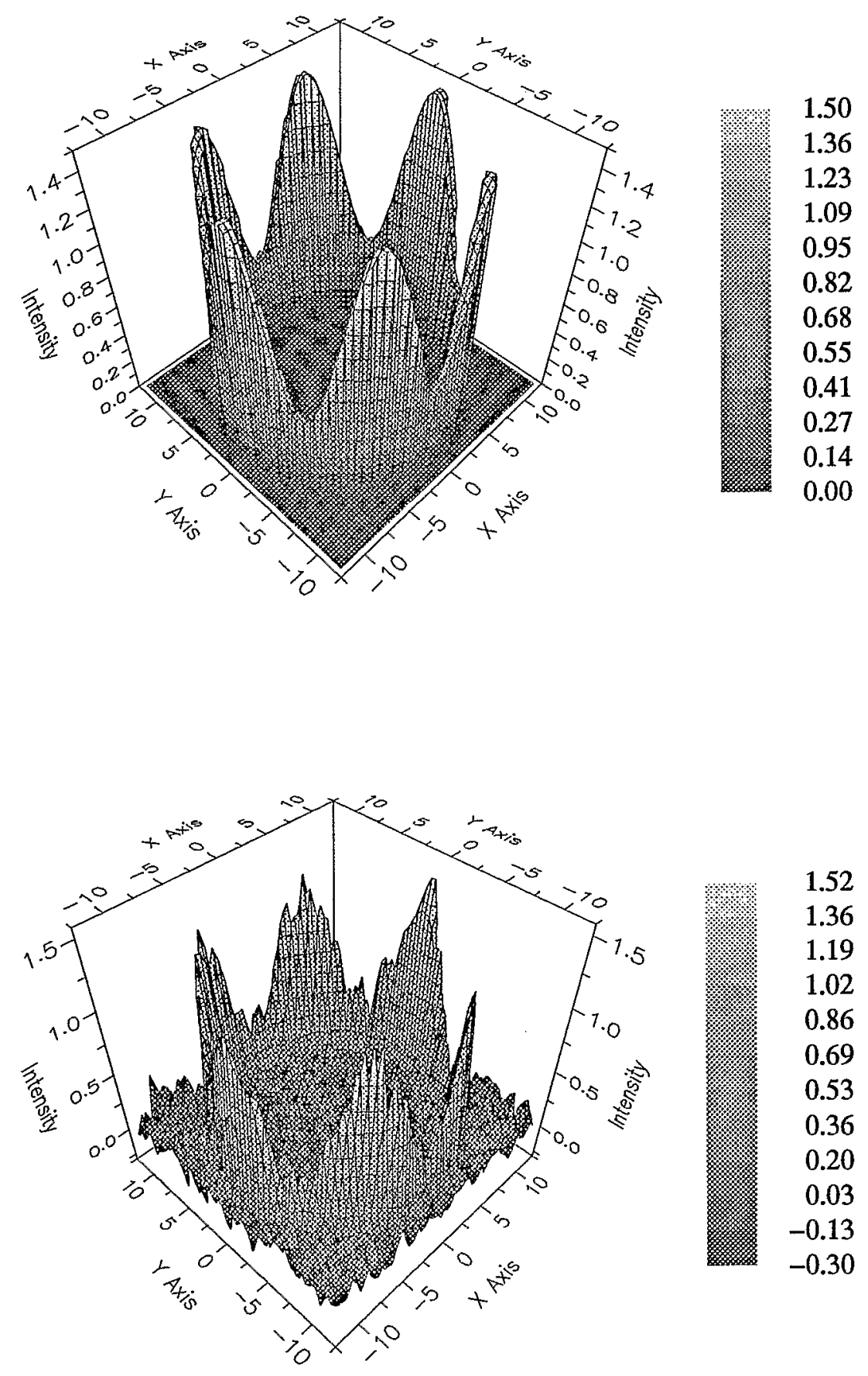

Figure 17: Reconstruction with noisy data. Original image (top) and reconstruction (bottom) using $t r c$, with hann filter. Total profiles $=30$, samples $=64$. Annular beam, azimuthal mode number $1=6$. 


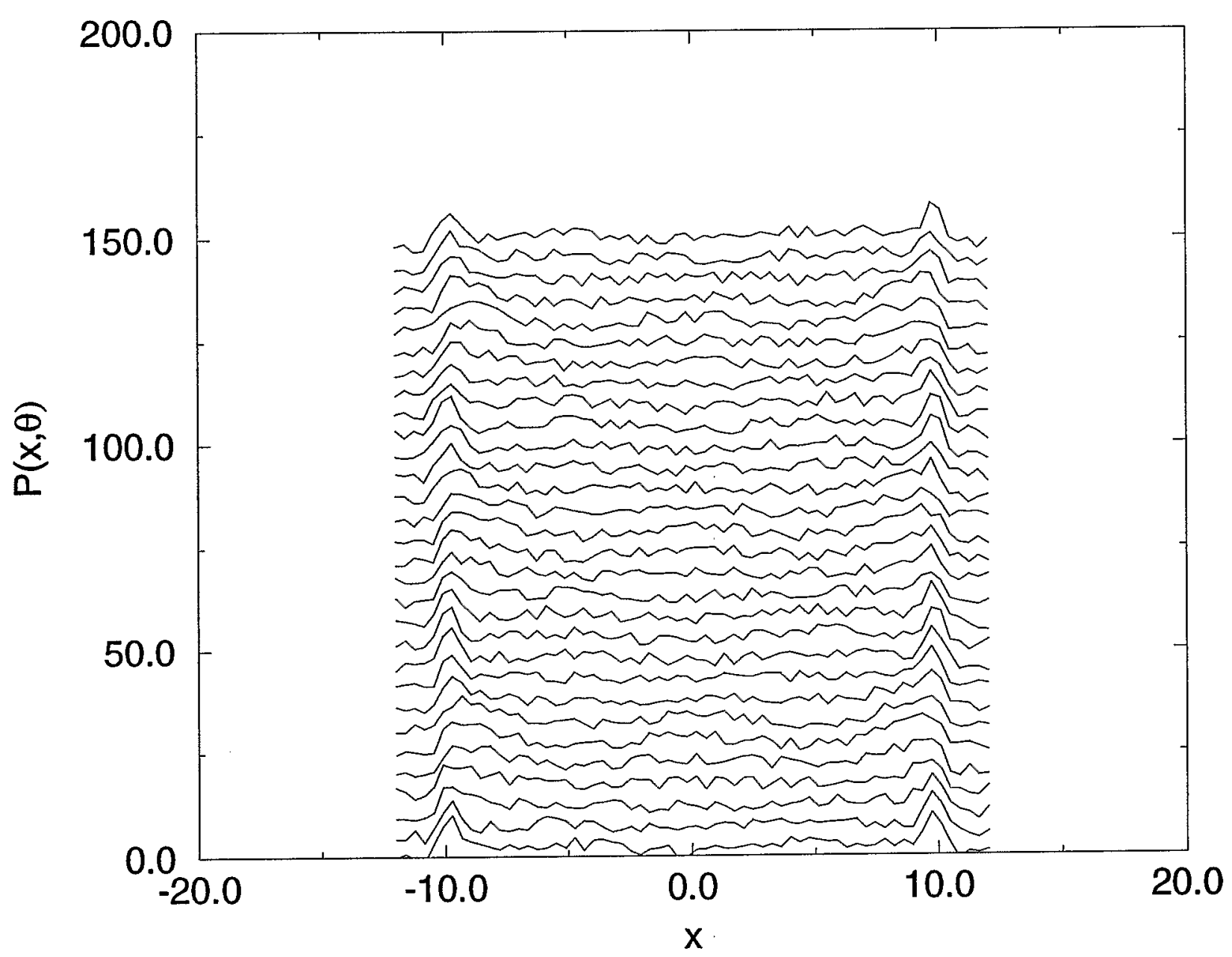

Figure 18: Input profiles for the reconstruction in figure 17. Total profiles $=30$. 


\section{Sensitivity Plots}

In order to determine the required number of profiles and samples per profile for good reconstruction, sensitivity plots are done for a guassian distribution. The parameters Signal Error and Noise Error, as described in Section 5 . are used to compare the recontructed image with the original image. The program image_comp is used to calculates these parameters for images of size $64 \times 64$. If the reconstructed image size is less than $64 \times 64$, it is interpolated using the program image_intr to size $64 \mathrm{x} 64$ and then compared with the original. The reconstructed image is compared with the original image for a guassian distribution. The image window extends to $+/-3 \sigma_{x}$ and $+/-7 \sigma_{y}$. In Figure 19 , the number of samples/profile varies from 4 through 128, and the number of profiles is fixed at 32 . When the number of samples is small (less than 32 ), the signal error is large (.2 to .4), but the background error is small, as the reconstructed image is close to zero. For good reconstruction, both the signal error and the background error should be small (less than .1).

With 32 samples, which is about 5 samples $/ \sigma_{x}$ and two samples $/ \sigma_{y}$ the signal error $E_{s}$ is .025 and the background error $E_{b}$ is .009 . The error does not decrease beyond this point. Therefore for a good reconstruction, there should be at least 3-5 samples/sigma.

Figure 20 shows the errors vs the number of profiles, which varies from 4 through 128 . The number of samples is fixed at 64 . When the number of profiles is 4, the signal error is .11 and the noise error is .11. The negative intensity is $27 \%$ of the maximum intensity. Therefore, this is not a good reconstruction as can be seen in the figure. When the number of profiles is 16 , both the signal and the noise error are less than .01 and remain flat beyond this point. For good reconstruction, the number of profiles should be at least 16. This is true for a smooth gaussian beam and no noise. With $20 \%$ random noise and 32 profiles the signal and background error are .08 . Therefore, with dipole motion and noise the number of profiles required is at least 32 .

Figure 21 shows the errors vs. the error in synchrotron period. The number of samples is 64 and the number of profiles in half synchrotron period is 64 . The number of profiles used for reconstruction varies from 16 through 112 . This gives an error in synchrotron period from $-75 \%$ to $75 \%$. When the number of profiles used is 32 out of $64(-50 \%$ error), both the signal and the background error are near their maximum as the reconstructed image is now rotated by 90 degree. For a good reconstruction (that is both signal and background error to be less than .10), the error in synchrotron period should be less than $15 \%$. 

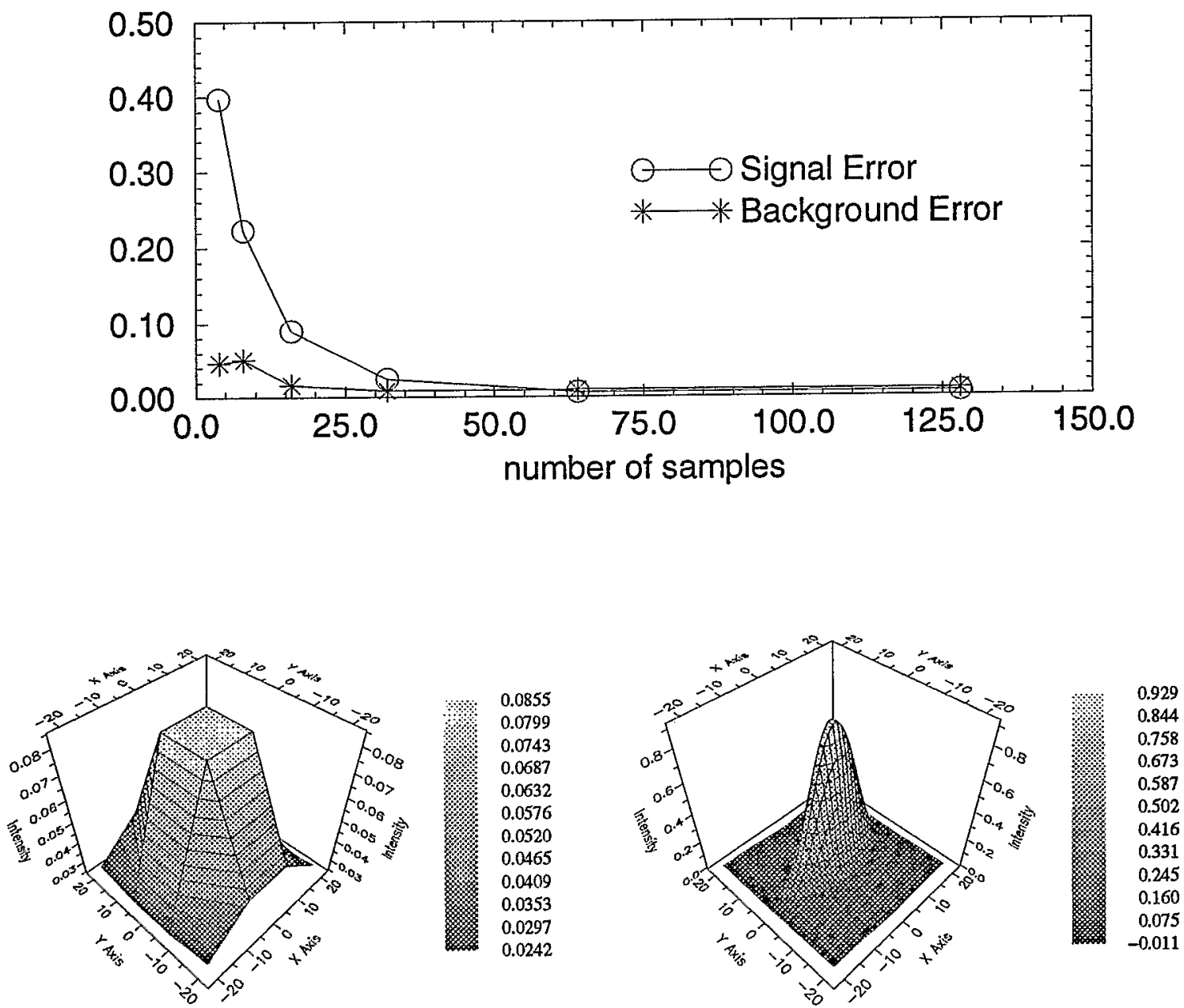

Figure 19: Error vs. number of samples for a gauss beam (top picture), total profiles $=32$, window $+/-3 \sigma_{x},+/-7 \sigma_{y}$. Reconstruction with 4 samples (bottom left) and 32 samples (bottom right). 

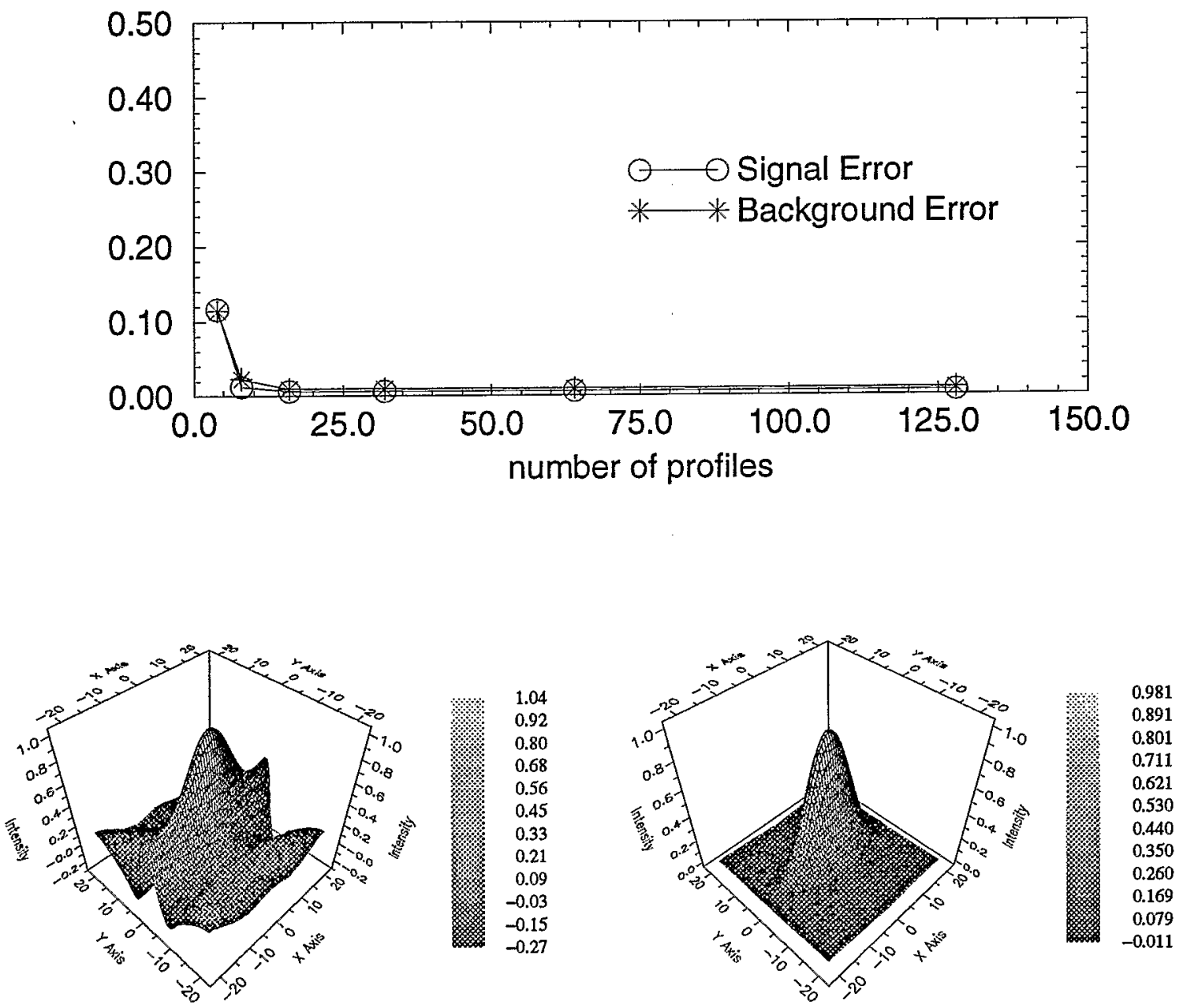

Figure 20: Error vs. number of profiles for a gauss beam (top picture), total samples $=64$, window $+/-3 \sigma_{x},+/-7 \sigma_{y}$. Reconstruction with 4 profiles (bottom left) and 16 profiles (bottom right). 

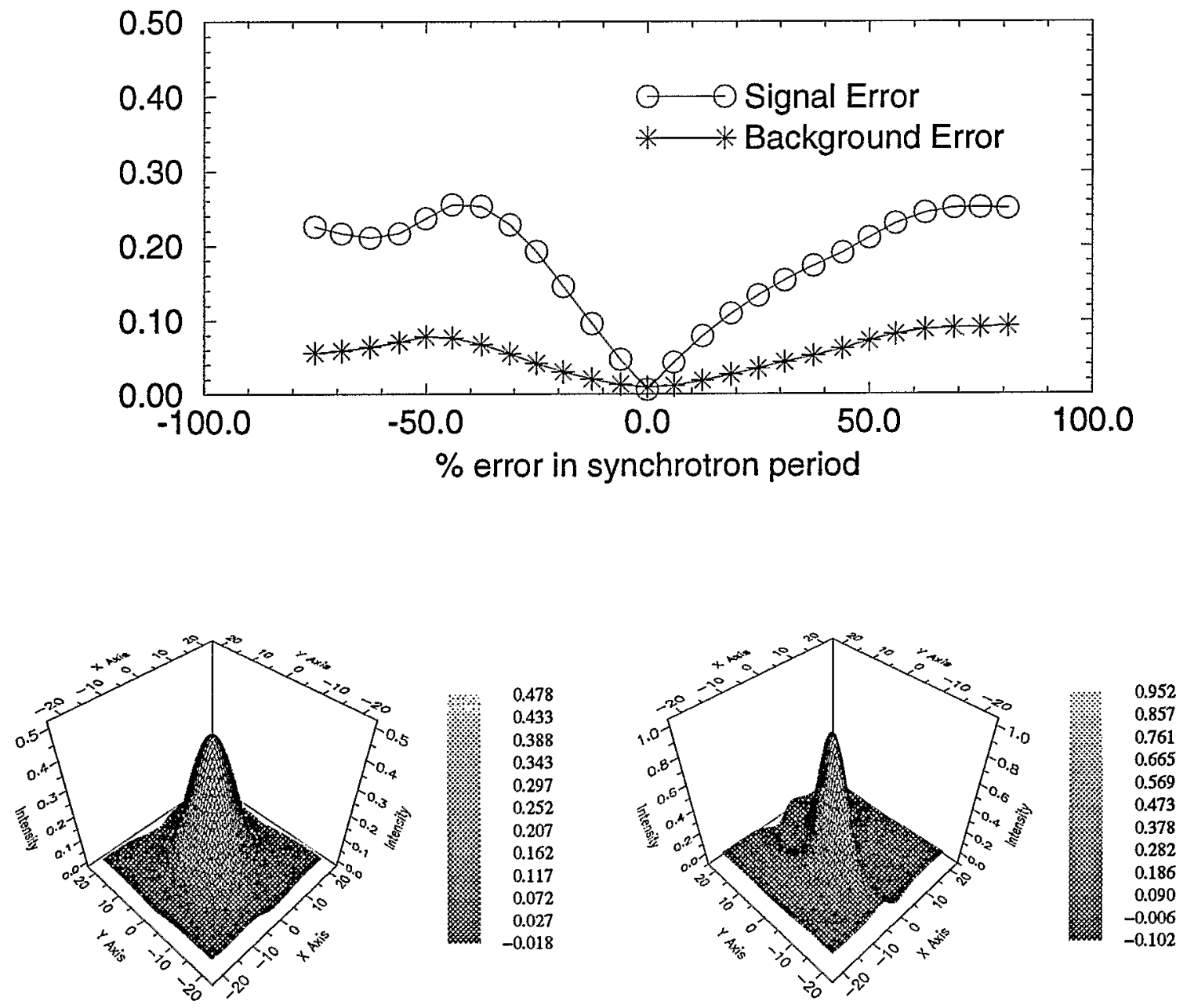

Figure 21: Error vs. error in synchrotron period for a gauss beam (top) total samples $=64$, total profiles $=64$, window $+/-3 \sigma_{x},+/-7 \sigma_{y} .16$ out of 64 profiles used, $-75 \%$ error (bottom left), and 32 out of 64 profiles used, $-50 \%$ error (bottom right). 


\section{TIBETAN Example}

tibetan [14] tracks a bunch of particles in longitudinal phase space, due to the influence of rf fields and the beam self fields. The reconstruction algorithms described above assume that the beam does not change shape in one synchrotron period. trc can be used at injection, top energy and acceleration provided the motion is adiabatic.

In the example described below, AGS injection parameters are used. $\gamma$ is $2.6, \gamma_{t}$ is 8.45 , harmonic number is 8 , rf frequency is $2.74 \mathrm{MHz}$, rf voltage is $40 \mathrm{kV}$, synchrotron period $\left(T_{s}\right)$ is $1.6 \mathrm{msec}$, and bunch area $\left(A_{b u n}\right)$ is $1.5 \mathrm{eV}$-sec. The number of particles used for tracking is 15000 . For reconstruction 55 profiles are used in half a synchrotron period, and there are 100 samples/profile. Figure 22 shows the beam distribution and the rf bucket from tibetan output, the input profiles and the beam reconstruction using trc. The reconstruction compares well with the output from tibetan.

\section{AGS Example}

Figure 23 gives an example of reconstruction with AGS data, Wall Current Monitor data is obtained using the LabVIEW Mountain Range Program [16]. The time interval between profiles is $100 \mu s e c$. There are about 35 profiles in half a synchrotron period, 180 samples/profile and the sampling interval is 1 nsec. The species used is proton and the AGS cycle time is $700 \mathrm{msec}$ (just above transition). $\gamma$ is $9.38, \gamma_{t}$ is 8.45 , harmonic number (h) is 8 , rf frequency is $2.95 \mathrm{MHz}$ rf voltage is $400 \mathrm{kV}, \phi_{s}$ is 2.85 and $\dot{B}$ is 1.66 $\mathrm{T} / \mathrm{sec}$. Synchrotron period $\left(T_{s}\right)$ is $7 \mathrm{msec}$ and the change in synchrotron period in half a synchrotron period (i.e. $3.5 \mathrm{msec}$ ) is less than $1 \%$.

\section{Conclusion and Status}

A program trc has been developed in $\mathrm{C}$ to do image reconstruction from profiles. The code can read mountain range data in sds format from tibetan. A code has also been written to convert the wall current monitor data from the LabVIEW program to sds format. Hence data flow from the wall current monitor to $t r c$ has been demonstrated. Reconstruction with $t r c$ has been illustrated with examples of gaussian and annular density distributions. Reconstruction in longitudinal phase space has been demonstrated with an example from tibetan, and an example from AGS data. More work on application of the code to real data from AGS has to be done.

\section{Acknowledgements}

The author would like to thank B. Andrews, R. Marr and J. Wei for helpful discussions, and W. Van Asselt for help with obtaining AGS data. 

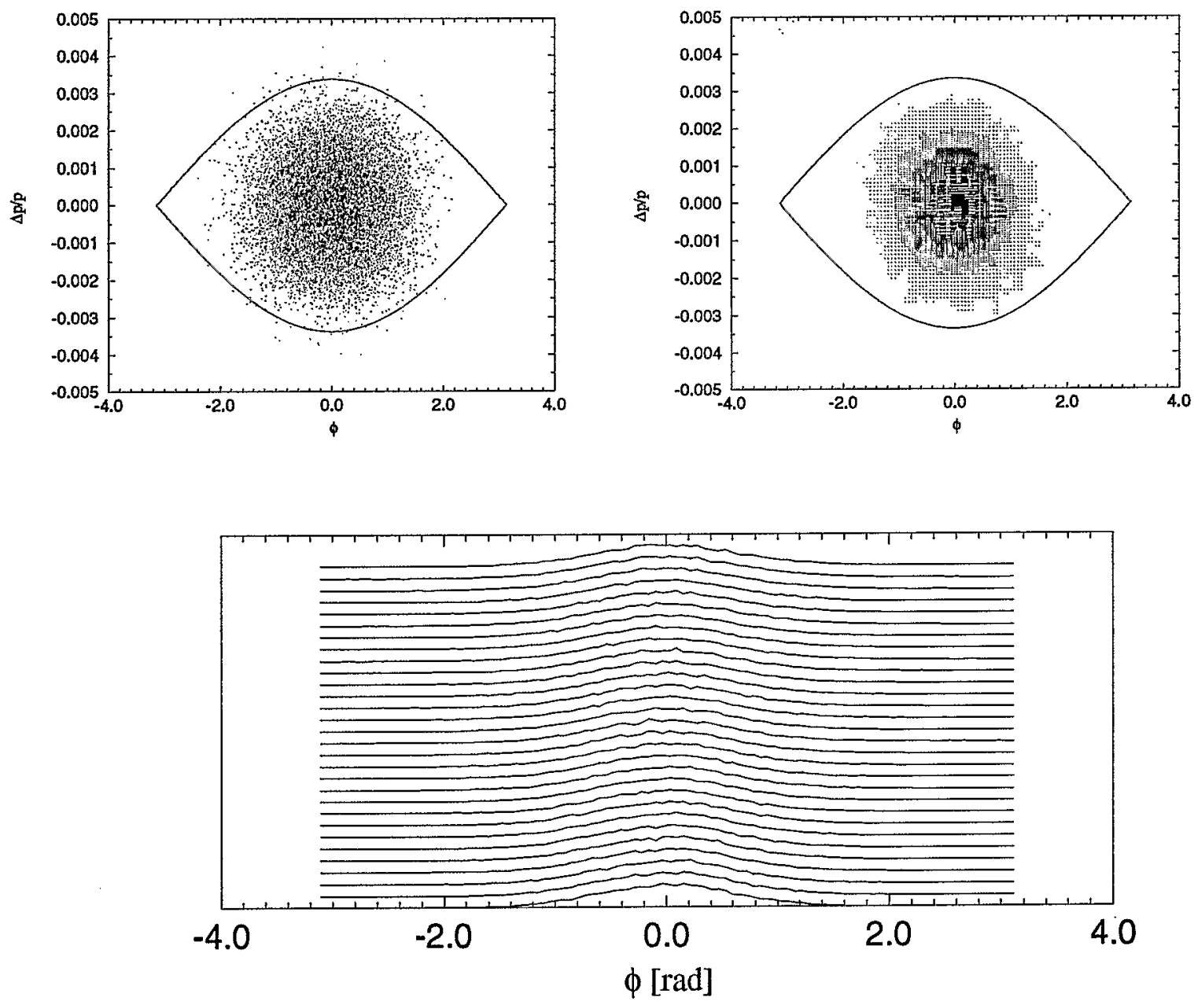

Figure 22: tibetan Simulation (top left), trc reconstruction (top right), and input profiles used in trc (bottom). Beam parameters are similar to AGS injection parameters. $\gamma=2.6, \gamma_{t}=8.5, \mathrm{~h}=8, f_{r f}=$ $2.74 \mathrm{MHz}, V_{r f}=40 \mathrm{kV}, T_{s}=1.6 \mathrm{msec}$ and $A_{b u n}=1.5 \mathrm{eV}$-sec, profiles $=55$, samples $=100$. 

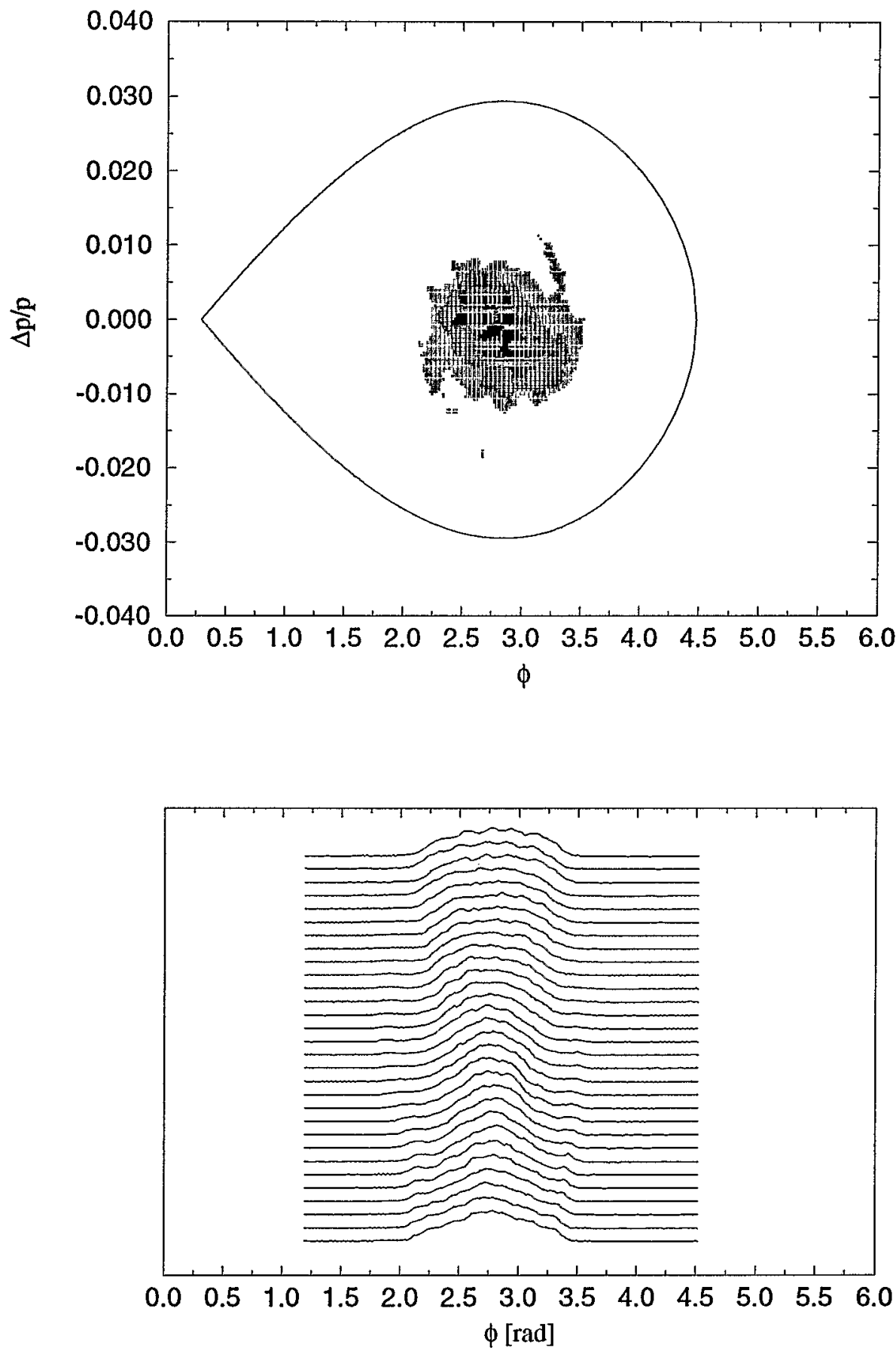

Figure 23: AGS data: Phase Space reconstruction with trc (top) and input mountain range data (bottom). Proton, $\gamma=9.38, \gamma_{t}=8.45$, harmonic number $=8, f_{r f}=2.95 \mathrm{MHz}, V_{r f}=400 \mathrm{kV} \phi_{s}=2.85 \mathrm{rad}, \dot{B}=$ $1.66 \mathrm{~T} / \mathrm{sec}, T_{s}=7 \mathrm{msec}$, profiles $=36$, samples $=180$. 


\section{References}

[1] Johann Radon, Ber. Verh. Sächs. Akad. Wiss. Leipzig, Math. Phys. K1. 69, 262-277 (1917).

[2] Stanley R. Deans, The Radon Transform and some of its applications, J. Wiley, 1983.

[3] H. H. Barrett, W. Swindell, Radiological Imaging, Academic Press, 1981.

[4] G. T. Herman, Image Reconstruction from Projections, Springer-Verlag, 1979.

[5] R. Gordon, R. Bender, G. T. Herman, J. Theor. Biol., (1970), 29, pp 471-481.

[6] R. Gordan, IEEE Trans. Nucl. Sci., Vol. NS-21, June 1974 pp 78-93

[7] G. T. Herman, J. Theor. Biol, (1973) 42, pp 1-32

[8] RECLBL, Donner Algorithms for Reconstruction Tomography, R. H. Huesman, G. T. Gullberg, W. L. Greenberg, T. F. Budinger, October 1977.

[9] SNARK93, J. A. Browne, G. T. Herman, D. Odhner, Tech. Rep No. MIPG198, August 1993.

[10] R.N.Bracewell, A.C.Riddle, Astrophys.J. 150 (2), pp 427-434 (1967).

[11] G.N.Ramachandran, A.V.Lakshminarayanan, Proc.Natl.Acad.Sci. 68 (9), pp 2236-2240 (1971).

[12] F. J. Sacherer, IEEE Trans. Nucl. Sci., Vol. NS-20, 825 (1973).

[13] C. Saltmarsh, RHIC AP Note 29, July 1994

[14] Jie Wei, PhD Thesis, SUNY, Stony Brook, May 1990.

[15] S. Tepikian, Flag Display Program.

[16] W. Van Asselt, LabVIEW Mountain Range Program.

[17] LabVIEW, National Instruments Corporation, 1992 